\title{
Low-Frequency Scaling of the Standard and Mixed Magnetic Field and Müller Integral Equations
}

\author{
Ignace Bogaert, Member, IEEE, Kristof Cools and Francesco P. Andriulli, Senior Member, IEEE \\ and Hakan Bağcı, Member, IEEE
}

\begin{abstract}
The standard and mixed discretizations for the Magnetic Field Integral Equation (MFIE) and the Müller Integral Equation (MUIE) are investigated in the context of lowfrequency (LF) scattering problems involving simply connected scatterers. It is proved that, at low frequencies, the frequency scaling of the nonsolenoidal part of the solution current can be incorrect for the standard discretization. In addition, it is proved that the frequency scaling obtained with the mixed discretization is correct. The reason for this problem in the standard discretization scheme is the absence of exact solenoidal currents in the rotated RWG finite element space. The adoption of the mixed discretization scheme eliminates this problem and leads to a well-conditioned system of linear equations that remains accurate at low frequencies. Numerical results confirm these theoretical predictions and also show that, when the frequency is lowered, a finer and finer mesh is required to keep the accuracy constant with the standard discretization.
\end{abstract}

Index Terms-Mixed Discretization, Magnetic Field Integral Equation, Müller Integral Equation, Accuracy, Low-Frequency Stability.

\section{INTRODUCTION}

I NTEGRAL equations of the first kind are widely used for the modeling of time-harmonic scattering problems, e.g. the Electric Field Integral Equation (EFIE) for Perfect Electric Conductors (PECs) and the Poggio-Miller-Chang-HarringtonWu-Tsai Integral Equation (PMIE) for dielectrics [1]. These equations are usually discretized using RWG basis functions [2] and rotated RWG testing functions, where 'rotated' means that the cross product with the surface normal was taken. This discretization results in what will be called the standard EFIE and standard PMIE in the rest of this paper. It is also possible to model these scattering problems using integral equations of the second kind. For the case of PECs, this equation is the MFIE [3], [4] while for dielectrics it is the MUIE [5]. The standard discretization strategy for the MFIE and MUIE is to use RWG basis and testing functions.

In general, integral equations of the second kind are less widely used than those of the first kind because they cannot be applied to open PEC scatterers (e.g. plates) and, when using the standard discretization strategy, they offer inferior

Ignace Bogaert is with the Department of Information Technology, Ghent University, Sint-Pietersnieuwstraat 41, B-9000 Gent, Belgium, e-mail: Ignace.Bogaert@intec.UGent.be.

Kristof Cools is with the Electrical Systems and Optics Research Division, University of Nottingham, Nottingham NG7 2RD, U.K.

Francesco Andriulli is with the Microwave Department of Telecom Bretagne - Institut Mines-Telecom, Brest, France.

Hakan Bağc1 is with the Division of Computer, Electrical, and Mathematical Sciences and Engineering, King Abdullah University of Science and Technology (KAUST), Thuwal, 23955-6900, Saudi Arabia. accuracy when compared to equations of the first kind [6]. Nevertheless, second kind integral equations are of great practical importance because of their role in the Combined Field Integral Equation (CFIE) and because they yield wellconditioned systems of linear equations without special preconditioning strategies (e.g. Calderón preconditioning [7]). With the advent of fast iterative solution methods [8]-[10], the condition number of the linear system has become more and more important, which has in turn made integral equations of the second kind more attractive.

Because of this, much research has been focused on solving the accuracy problem of integral equations of the second kind. These efforts include increasing the accuracy of the MFIE matrix elements [11], [12], incorporating a solid angle contribution [13] or enriching the finite element space [14], [15]. The aforementioned techniques have been demonstrated to result in a significant accuracy improvement when applied to certain classes of scattering problems. However, they lack a clear theoretical background and it is hard to predict which method is best for a given situation. Recently, an alternative testing scheme for second kind integral equations was proposed in [16], [17] as a novel way to improve the accuracy. In this scheme, the equation is tested with rotated Buffa-Christiansen (BC) or Chen-Wilton (CW) functions [18], [19] instead of RWG functions. Since the test functions are no longer the rotated basis functions, this scheme is usually called a mixed discretization. The mixed scheme is convergent and numerical results show that it yields a significant accuracy improvement when compared to the standard testing scheme, even when applied to an object with sharp corners such as a cube [17].

In this paper, the focus is on the LF behavior of the solution currents. From [20], it is known that the inferior accuracy of the standard discretization scheme of the MFIE gets worse as the scatterer gets electrically smaller, i.e. as the frequency is lowered. This is caused by the fact that the magnetic field integral equation at very low frequencies leads to a large relative error in the nonsolenoidal part of the solution current. This means that the MFIE in its most basic form has very limited use at low frequencies. In the same paper [20], a method was proposed to solve this problem, which relies on manually setting the nonsolenoidal part of the current to zero in the static limit. However, this perturbation method has some computational drawbacks and can no longer be regarded as a straightforward Petrov-Galerkin discretization [21] of the MFIE. In [22] and [23], a current-charge formulation is proposed that imposes both the tangential and normal boundary conditions for the magnetic and electric field respectively. This 
increases the number of unknowns but allows the charge to be computed accurately, even for very low frequencies. In [24], it was shown that, for simply connected geometries and for plane wave incidence, the mixed discretization of the MFIE yields the correct nonsolenoidal part without introducing additional uknowns. Further preliminary results pertaining to the LF behavior of the mixed discretization of the MFIE have been presented in [24]-[29].

In this paper, an extension is made to the MUIE and to much more general incoming fields. The analysis is limited to simply connected scatterers. The multiply connected case is considerably more involved and is beyond the scope of this paper. First, the LF properties of the relevant integral operators and the incoming fields will be given in Sections II and III. In Sections V and VI, it will then be shown that the mixed discretization of the MFIE and MUIE leads to a correct frequency scaling for both the solenoidal and nonsolenoidal part of the solution current. The correctness is tested by comparing these frequency scalings with the scalings obtained using the standard EFIE and PMIE respectively. In the case of PEC scattering, a theoretical analysis of the continuous EFIE and MFIE also confirms these scalings. Finally, numerical results are presented that corroborate the theoretical results.

To unambiguously specify which discretization scheme is used, an integral equation of the second kind that is tested with RWG functions will be denoted as 'standard', as opposed to 'mixed' when it is tested with rotated BC functions. When neither 'standard' nor 'mixed' are specified, this denotes the continuous integral equation. Finally, an $e^{j \omega t}$ time dependence is assumed and suppressed.

\section{LF BEHAVIOR OF INTEGRAL OPERATORS}

To express the MFIE and MUIE later on, it is convenient to first introduce a succinct notation for the relevant integral operators. Consider a region $\Omega$ with a simply connected boundary $\Gamma$. Let $\hat{\boldsymbol{n}}(\boldsymbol{r})$ be the exterior surface normal to $\Gamma$, which is well-defined for all $r \in \Gamma$. Then define

$$
\mathcal{T}_{k}[\boldsymbol{j}](\boldsymbol{r})=-j k \hat{\boldsymbol{n}}(\boldsymbol{r}) \times \mathrm{P} . \mathrm{V} . \int_{\Gamma}\left[\left(\mathbb{1}+\frac{\nabla \nabla}{k^{2}}\right) G_{k}\left(\boldsymbol{r}-\boldsymbol{r}^{\prime}\right)\right] \cdot \boldsymbol{j}\left(\boldsymbol{r}^{\prime}\right) \mathrm{d}
$$

$\mathcal{K}_{k}[\boldsymbol{j}](\boldsymbol{r})=\hat{\boldsymbol{n}}(\boldsymbol{r}) \times \int_{\Gamma} \nabla G_{k}\left(\boldsymbol{r}-\boldsymbol{r}^{\prime}\right) \times \boldsymbol{j}\left(\boldsymbol{r}^{\prime}\right) \mathrm{d} S^{\prime}$,

where $\mathbb{1}$ is the 3 by 3 unit matrix and the Green function is given by

$$
G_{k}(\boldsymbol{r})=\frac{e^{-j k\|\boldsymbol{r}\|}}{4 \pi\|\boldsymbol{r}\|}
$$

The wave number $k$ is given by $k=\omega \sqrt{\varepsilon \mu}$ where $\omega$ is the pulsation, i.e. $2 \pi$ times the frequency. The parameters $\varepsilon$ and $\mu$ are the permittivity and permeability of the medium in which the operators are defined. In both (1) and (2) the evaluation point $r$ should always be on the boundary $\Gamma$, to ensure that the surface normal $\hat{\boldsymbol{n}}(\boldsymbol{r})$ is well-defined. It should be noted that operator (2) is not a limiting value from either inside or outside of $\Omega$. Rather it is the average of these two limits. To get the inner or outer limiting value, an additional operator, proportional to the identity, has to be added. This identity operator will be denoted as

$$
\mathcal{I}[\boldsymbol{j}](\boldsymbol{r})=\boldsymbol{j}(\boldsymbol{r})
$$

For the rest of the paper, it is convenient to introduce a mild abuse of notation by removing the explicit dependence of the operators on the coordinates and allowing a matrix notation. For example when $\boldsymbol{t} \cdot \mathcal{K}_{k} \cdot \boldsymbol{b}$ is written, this actually means

$$
\int_{\Gamma} \boldsymbol{t}(\boldsymbol{r}) \cdot \mathcal{K}_{k}[\boldsymbol{b}](\boldsymbol{r}) \mathrm{d} S
$$

The operators given in the above are all endowed with specific frequency scaling properties when they are applied to solenoidal surface currents, i.e. divergence-free currents defined on the boundary $\Gamma$, at low frequencies. These properties are required for the analysis of integral equations at low frequencies and will now be briefly summarized. To denote that a quantity $\mathrm{V}$ scales like $\omega^{p}$ the following notation will be used

$$
\mathrm{V}=\mathcal{O}\left(\omega^{p}\right)
$$

which has the mathematical meaning

$$
\left|\lim _{\omega \rightarrow 0} \omega^{-p} \bigvee\right|<\infty
$$

Note that this definition does not completely fix the frequency scaling. Indeed, if (6) holds, then $\mathrm{V}=\mathcal{O}\left(\omega^{p^{\prime}}\right)$ also holds if $p^{\prime} \leq p$. This reasoning also shows that the statement with $p$ is stronger, i.e. contains more information, than the one with $p^{\prime}$. Therefore, statements like (6) in this paper will be intended to give the strongest scaling that can be proved for a general geometry, complex permittivity, etcetera.

The $\mathcal{K}_{k}$ operator (2) has the following property: if both $\boldsymbol{t}(\boldsymbol{r})$ and $\boldsymbol{b}(\boldsymbol{r})$ are solenoidal currents, then

$$
[\hat{\boldsymbol{n}} \times \boldsymbol{t}] \cdot \mathcal{K}_{k} \cdot \boldsymbol{b}(\boldsymbol{r})=\mathcal{O}\left(\omega^{2}\right)
$$

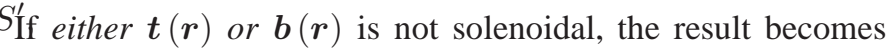
$\mathcal{O}(1)$. This property has been first derived in [30]. A related property can be found for the identity operator: if both $\boldsymbol{t}(\boldsymbol{r})$ and $\boldsymbol{b}(\boldsymbol{r})$ are solenoidal currents, then

$$
[\hat{\boldsymbol{n}} \times \boldsymbol{t}] \cdot \mathcal{I}_{k} \cdot \boldsymbol{b}(\boldsymbol{r})=0 .
$$

Otherwise the result is again $\mathcal{O}(1)$. For a proof, we refer to [31]. Integral operator (1) has a very well-known behavior at low frequencies that is used in the loop star decomposition for the EFIE [32]. If either $\boldsymbol{t}(\boldsymbol{r})$ or $\boldsymbol{b}(\boldsymbol{r})$ is a solenoidal current

$$
[\hat{\boldsymbol{n}} \times \boldsymbol{t}] \cdot \mathcal{T}_{k} \cdot \boldsymbol{b}(\boldsymbol{r})=\mathcal{O}(\omega) .
$$

Otherwise, the result is $\mathcal{O}\left(\frac{1}{\omega}\right)$. In addition, whatever $\boldsymbol{t}(\boldsymbol{r})$ and $\boldsymbol{b}(\boldsymbol{r})$ are

$$
[\hat{\boldsymbol{n}} \times \boldsymbol{t}] \cdot\left[k_{1} \mathcal{T}_{k_{1}}-k_{2} \mathcal{T}_{k_{2}}\right] \cdot \boldsymbol{b}(\boldsymbol{r})=\mathcal{O}\left(\omega^{2}\right),
$$

always holds. A proof of this property can be found in [33]. 


\section{LF BEHAVIOR OF INCOMING FIELDS}

Let the incoming electric and magnetic fields be denoted by $\boldsymbol{e}_{i}(\omega, \boldsymbol{r})$ and $\boldsymbol{h}_{i}(\omega, \boldsymbol{r})$. Assume that these incoming fields are generated by a collection of electric and magnetic currents, located in a region $S$ outside of $\Omega$ and denoted by $\boldsymbol{j}_{i}(\omega, \boldsymbol{r})$ and $\boldsymbol{m}_{i}(\omega, \boldsymbol{r})$ respectively. Note that this assumption is without loss of generality since fields generated by sources at infinity (such as a plane wave) can also be generated by equivalent currents at finite positions. For the purposes of this paper, it will be assumed that $\boldsymbol{j}_{i}(\omega, \boldsymbol{r})$ and $\boldsymbol{m}_{i}(\omega, \boldsymbol{r})$ are analytic functions of $\omega$ in a neighborhood containing $\omega=0$. Although it is definitely possible to find currents that violate this constraint, the overwhelming majority of real-world currents are analytic as a function of $\omega$. For example, any timelimited or exponentially decaying time domain current has an analytic frequency domain counterpart. Also, should the need arise, the calculations in this paper are readily generalized to arbitrary frequency dependencies for the currents. This generalization results in lengthier expressions, but does not require the introduction of new theory.

Now, assuming the currents are indeed analytic functions of $\omega$, a Taylor series argument shows that for low frequencies, the currents scale as some positive integer power of $\omega$

$$
\begin{gathered}
\boldsymbol{j}_{i}(\omega, \boldsymbol{r})=\mathcal{O}\left(\omega^{\alpha_{e}}\right), \boldsymbol{m}_{i}(\omega, \boldsymbol{r})=\mathcal{O}\left(\omega^{\alpha_{m}}\right), \\
\alpha_{t} \in \mathbb{N}, \forall t \in\{e, m\}
\end{gathered}
$$

In many important cases, such as the equivalent currents generating a plane wave, the exponents $\alpha_{t}$ are zero, i.e. the current has a DC component. However, for the sake of generality, $\alpha_{t}$ will be left unspecified in the upcoming analysis.

The behavior of the charges associated with the currents can be investigated by means of

$$
\rho_{i}(\omega, \boldsymbol{r})=\frac{j}{\omega} \nabla \cdot \boldsymbol{j}_{i}(\omega, \boldsymbol{r}), \pi_{i}(\omega, \boldsymbol{r})=\frac{j}{\omega} \nabla \cdot \boldsymbol{m}_{i}(\omega, \boldsymbol{r}) .
$$

Because of the factor $\frac{1}{\omega}$, the charges can become infinite at $\omega \approx 0$. However, such a singularity does not make a charge distribution spurious or less important for simulations. For example, they can occur when doing a series of frequency domain simulations to construct a time domain simulation. Indeed, if the time domain excitation current leaves behind a nonzero static charge distribution once it has stopped flowing, the resulting frequency domain charge has a $\frac{1}{\omega}$ singularity. Therefore, charges with a singular $\frac{1}{\omega}$ dependence will be allowed in the rest of this paper. In general, the LF behavior of the charge can be described as

$$
\begin{gathered}
\rho_{i}(\omega, \boldsymbol{r})=\mathcal{O}\left(\omega^{\beta_{e}}\right), \pi_{i}(\omega, \boldsymbol{r})=\mathcal{O}\left(\omega^{\beta_{m}}\right), \\
\beta_{t} \in \mathbb{N}, \forall t \in\{e, m\}
\end{gathered}
$$

where $\beta_{t} \geq \alpha_{t}-1$ should hold for compatibility with (13).

With the knowledge of the LF behavior of the currents and charges, it becomes possible to investigate the behavior of the incoming electric and magnetic fields when they are integrated with solenoidal currents. For example, take the case of the incoming electric field, generated by the electric current $\boldsymbol{j}_{i}(\omega, \boldsymbol{r})$. When this field is integrated with the solenoidal current $t(r)$, the scaling of the result is

$$
\begin{aligned}
\int_{\Gamma} \boldsymbol{e}_{i}(\omega, \boldsymbol{r}) \cdot \boldsymbol{t}(\boldsymbol{r}) \mathrm{d} S & =\frac{\omega \mu}{j} \iint_{\Gamma} G_{k}\left(\boldsymbol{r}-\boldsymbol{r}^{\prime}\right) \boldsymbol{j}_{i}\left(\omega, \boldsymbol{r}^{\prime}\right) \cdot \boldsymbol{t}(\boldsymbol{r}) \mathrm{d} S \mathrm{~d} \boldsymbol{r}^{\prime} \\
& =\mathcal{O}\left(\omega^{\alpha_{e}+1}\right) .
\end{aligned}
$$

In the above, only the vector potential term of the Green's dyadic remains because $\boldsymbol{t}(\boldsymbol{r})$ is solenoidal.

When the magnetic field generated by the electric current is integrated with the solenoidal current, the following is obtained

$$
\begin{aligned}
\int_{\Gamma} \boldsymbol{h}_{i}(\omega, \boldsymbol{r}) \cdot \boldsymbol{t}(\boldsymbol{r}) \mathrm{d} S & =j \omega \epsilon \int_{\Gamma} \boldsymbol{e}_{i}(\omega, \boldsymbol{r}) \cdot \hat{\boldsymbol{n}}(\boldsymbol{r}) \phi(\boldsymbol{r}) \mathrm{d} S \\
& =\mathcal{O}\left(\omega^{\min \left(\alpha_{e}+2, \beta_{e}+1\right)}\right) .
\end{aligned}
$$

Here, the scalar function $\phi(r)$ is chosen such that

$$
\boldsymbol{t}(\boldsymbol{r})=\hat{\boldsymbol{n}}(\boldsymbol{r}) \times \nabla \phi(\boldsymbol{r}) .
$$

This allows a reasoning similar to equation (37) in [34], to convert the incoming magnetic field into the incoming electric field, as is done in (16).

\section{DisCRETIZATION}

The boundary $\Gamma$ is discretized using a simplicial mesh consisting of $V$ vertices, $F$ faces and $E$ edges. Because $\Gamma$ is simply connected, it follows that $E=F+V-2$. Throughout the paper, the RWG and BC functions will be denoted by $\boldsymbol{f}_{n}(\boldsymbol{r})$ and $\boldsymbol{g}_{n}(\boldsymbol{r})$ respectively for all $n \in[1, E]$.

As is well-known, the RWG and BC finite element spaces can both be split up into a solenoidal and complementary nonsolenoidal subspace [20], [31], [32], [35]. The dimension of the RWG solenoidal subspace is $V-1$ and that of the nonsolenoidal subspace is $F-1$. For the BC finite element space, the roles of vertices and faces are reversed, which leads to the dimension of the $\mathrm{BC}$ nonsolenoidal space $V-1$ and the dimension of the $\mathrm{BC}$ solenoidal space $F-1$.

In the rest of the paper, elements of the RWG and BC solenoidal spaces will be denoted by $\boldsymbol{f}_{n}^{l}(\boldsymbol{r}) \forall n \in[1, V-1]$ and $\boldsymbol{g}_{n}^{l}(\boldsymbol{r}) \forall n \in[1, F-1]$ respectively ( $l$ stands for loop), while the nonsolenoidal spaces will be denoted by $\boldsymbol{f}_{n}^{s}(\boldsymbol{r}) \forall n \in[1, F-1]$ and $\boldsymbol{g}_{n}^{s}(\boldsymbol{r}) \forall n \in[1, V-1]$ respectively ( $s$ stands for star).

\section{LF BEHAVIOR OF THE MFIE}

When $\Omega$ is filled with a perfectly conducting medium and embedded in a background medium with permittivity $\varepsilon_{o}$, permeability $\mu_{o}$, wave number $k_{o}=\omega \sqrt{\varepsilon_{o} \mu_{o}}$ and impedance $\eta_{o}=\sqrt{\frac{\mu_{o}}{\varepsilon_{o}}}$, the MFIE is the most commonly used integral equation of the second kind. It is given by

$$
\frac{1}{2} \boldsymbol{j}-\mathcal{K}_{k_{o}} \cdot \boldsymbol{j}=\hat{\boldsymbol{n}} \times \boldsymbol{h}^{i},
$$

where $\boldsymbol{j}(\boldsymbol{r})$ is the current distribution to be solved for. In both the standard and mixed discretization of the MFIE, the unknown current distribution $\boldsymbol{j}(\boldsymbol{r})$ is expanded in RWGs. To obtain the standard MFIE, equation (18) is tested with RWG functions. In the mixed MFIE, on the other hand, rotated BC 
functions are used as testing functions. However, to expose the LF behavior of the standard and mixed MFIE, it is necessary to perform a basis transformation from the RWG space into its decomposition in solenoidal and nonsolenoidal currents. This is equivalent to expanding the unknown solution current into RWG solenoidal and nonsolenoidal functions

$$
\boldsymbol{j}_{\boldsymbol{t}}(\boldsymbol{r})=\sum_{m=1}^{V-1}\left[\boldsymbol{a}_{\boldsymbol{t}}^{l}\right]_{m} \boldsymbol{f}_{m}^{l}(\boldsymbol{r})+\sum_{m=1}^{F-1}\left[\boldsymbol{a}_{\boldsymbol{t}}^{s}\right]_{m} \boldsymbol{f}_{m}^{s}(\boldsymbol{r}) .
$$

The index $\boldsymbol{t}$ of the unknown current denotes the type of test function used, i.e. $\boldsymbol{f}$ for RWGs or $\hat{\boldsymbol{n}} \times \boldsymbol{g}$ for rotated BCs. When these test function spaces are also split into solenoidal and nonsolenoidal subspaces, the discretized MFIE can finally be written in block matrix form

$$
\left[\begin{array}{ll}
\mathrm{M}_{t}^{l l} & \mathrm{M}_{t}^{l s} \\
\mathrm{M}_{t}^{s l} & \mathrm{M}_{t}^{s s}
\end{array}\right] \cdot\left[\begin{array}{l}
\boldsymbol{a}_{t}^{l} \\
\boldsymbol{a}_{t}^{s}
\end{array}\right]=\left[\begin{array}{l}
\boldsymbol{h}_{t}^{l} \\
\boldsymbol{h}_{t}^{s}
\end{array}\right]
$$

with

$$
\begin{aligned}
{\left[\mathrm{M}_{\boldsymbol{t}}^{p q}\right]_{n m} } & =\boldsymbol{t}_{n}^{p} \cdot\left[\frac{1}{2} \boldsymbol{f}_{m}^{q}-\mathcal{K}_{k_{o}} \cdot \boldsymbol{f}_{m}^{q}\right], \\
{\left[\boldsymbol{h}_{\boldsymbol{t}}^{p}\right]_{n} } & =\boldsymbol{t}_{n}^{p} \cdot\left[\hat{\boldsymbol{n}} \times \boldsymbol{h}^{i}\right] .
\end{aligned}
$$

It should be stressed that the solution current obtained after solving (20) is exactly the same (up to direct or iterative solver accuracy, of course) as the current obtained from the direct discretization, i.e. without the decomposition into solenoidal and nonsolenoidal currents. Indeed, up to a basis transformation, the linear equations are the same. Therefore, no quasi-Helmholtz decomposition of the finite element spaces is needed when solving for the current, and it is done here solely for the purpose of showing the frequency dependence of the solenoidal and nonsolenoidal parts of the solution current. For the MFIE, avoiding the quasi-Helmholtz decomposition is advantageous because of its inherent well-conditioned behavior.

The mixed MFIE will be investigated first. Due to (8) and (9), it is concluded that

$$
\left[\begin{array}{ll}
\mathrm{M}_{\hat{\boldsymbol{n}} \times \boldsymbol{g}}^{l l} & \mathrm{M}_{\hat{\boldsymbol{n}} \times \boldsymbol{g}}^{l s} \\
\mathrm{M}_{\hat{\boldsymbol{n}} \times \boldsymbol{g}}^{s l} & \mathrm{M}_{\hat{\boldsymbol{n}} \times \boldsymbol{g}}^{s s}
\end{array}\right]=\left[\begin{array}{cc}
\mathcal{O}\left(\omega^{2}\right) & \mathcal{O}(1) \\
\mathcal{O}(1) & \mathcal{O}(1)
\end{array}\right] .
$$

Assuming that the mixed MFIE is invertible, it immediately becomes clear that the square blocks $\mathrm{M}_{\hat{\boldsymbol{n}} \times \boldsymbol{g}}^{s l}$ and $\mathrm{M}_{\hat{\boldsymbol{n}} \times \boldsymbol{g}}^{l s}$ must also be invertible at sufficiently low frequencies. Using this knowledge, a reasoning based on the Schur complement [36] of these blocks, detailed in Appendix A, shows that

$$
\left[\begin{array}{cc}
\mathrm{M}_{\hat{\boldsymbol{n}} \times \boldsymbol{g}}^{l l} & \mathrm{M}_{\hat{\boldsymbol{n}} \times \boldsymbol{g}}^{l s} \\
\mathrm{M}_{\hat{\boldsymbol{n}} \times \boldsymbol{g}}^{s l} & \mathrm{M}_{\hat{\boldsymbol{n}} \times \boldsymbol{g}}^{s s}
\end{array}\right]^{-1}=\left[\begin{array}{cc}
\mathcal{O}(1) & \mathcal{O}(1) \\
\mathcal{O}(1) & \mathcal{O}\left(\omega^{2}\right)
\end{array}\right] .
$$

If the incoming field is generated by a superposition of electric and magnetic currents, then with the scalings introduced in Section III

$$
\begin{aligned}
& {\left[\boldsymbol{h}_{\hat{\boldsymbol{n}} \times \boldsymbol{g}}^{l}\right]_{n}=\mathcal{O}\left(\omega^{\min \left(\alpha_{e}+2, \beta_{e}+1, \alpha_{m}+1\right)}\right),} \\
& {\left[\boldsymbol{h}_{\hat{\boldsymbol{n}} \times \boldsymbol{g}}^{s}\right]_{n}=\mathcal{O}\left(\omega^{\min \left(\alpha_{e}, \beta_{m}, \alpha_{m}+1\right)}\right) .}
\end{aligned}
$$

The fact that the $\mathrm{BC}$ finite element space contains a subspace of exact solenoidal currents is crucial for the first scaling result.
The second scaling is directly derived from the frequency scaling of the excitation currents (12). With these results, the frequency scaling of the solution of (20) can be obtained

$$
\begin{aligned}
{\left[\begin{array}{l}
\boldsymbol{a}_{\hat{\boldsymbol{n}} \times \boldsymbol{g}}^{l} \\
\boldsymbol{a}_{\hat{\boldsymbol{n}} \times \boldsymbol{g}}^{s}
\end{array}\right] } & =\left[\begin{array}{cc}
\mathcal{O}(1) & \mathcal{O}(1) \\
\mathcal{O}(1) & \mathcal{O}\left(\omega^{2}\right)
\end{array}\right] \cdot\left[\begin{array}{c}
\mathcal{O}\left(\omega^{\min \left(\alpha_{e}+2, \beta_{e}+1, \alpha_{m}+1\right)}\right) \\
\mathcal{O}\left(\omega^{\min \left(\alpha_{e}, \beta_{m}, \alpha_{m}+1\right)}\right)
\end{array}\right] \\
& =\left[\begin{array}{c}
\mathcal{O}\left(\omega^{\min \left(\alpha_{e}, \beta_{m}, \alpha_{m}+1\right)}\right) \\
\mathcal{O}\left(\omega^{\min \left(\alpha_{e}+2, \beta_{e}+1, \alpha_{m}+1\right)}\right)
\end{array}\right]
\end{aligned}
$$

It is interesting to point out that the fact that $\mathrm{M}_{\hat{\boldsymbol{n}} \times \boldsymbol{g}}^{s l}$ and $\mathrm{M}_{\hat{\boldsymbol{n}} \times \boldsymbol{g}}^{l s}$ are square is necessary for the Schur complement analysis. If the rotated $\mathrm{BC}$ functions were to be replaced with rotated RWG functions, the discretization scheme would still be conforming. However, the two off-diagonal blocks would no longer be square, which would make the discretized equation exactly singular for $\omega=0$. This may be interpreted as an explanation for why this discretization of the MFIE leads to ill-conditioned systems of linear equations.

For the standard MFIE, no exact solenoidal currents are present in the rotated RWG space. Hence none of the properties from section II can be used. Therefore

$$
\boldsymbol{a}_{\boldsymbol{f}}^{l}=\mathcal{O}\left(\omega^{\min \left(\alpha_{e}, \beta_{m}, \alpha_{m}+1\right)}\right)=\boldsymbol{a}_{\boldsymbol{f}}^{s} .
$$

Clearly, the standard MFIE yields different current scalings than the mixed MFIE. The question then becomes: which scaling is correct, if any ? A simple example shows that the scaling of the standard MFIE solution current is wrong in at least some cases: take a purely electric excitation current with $\alpha_{e}=0$ and $\beta_{e}=0$. This is an excitation current that generates a static electric and magnetic field at $\omega=0$. In this case, the standard MFIE solution current has an $\mathcal{O}(1)$ scaling for both the solenoidal and nonsolenoidal part. This means that the charge associated with the standard MFIE solution current diverges like $\frac{1}{\omega}$ as the frequency is lowered to zero. This diverging behavior is not consistent with reality, in which one would expect that the $\mathcal{O}(1)$ electric field induces an $\mathcal{O}(1)$ charge on the surface $\Gamma$. To investigate which of the scalings derived in the above is correct, a comparison with the scalings obtained from the discretized EFIE will be made. To further back up these results, a theoretical comparison with the continuous MFIE and EFIE will be made.

\section{A. Comparison with the standard EFIE}

The standard EFIE system matrix, written in block form, is

$$
\left[\begin{array}{cc}
\mathrm{E}_{f}^{l l} & \mathrm{E}_{f}^{l s} \\
\mathrm{E}_{\boldsymbol{f}}^{s l} & \mathrm{E}_{\boldsymbol{f}}^{s s}
\end{array}\right] \cdot\left[\begin{array}{c}
\boldsymbol{b}_{\boldsymbol{f}}^{l} \\
\boldsymbol{b}_{f}^{s}
\end{array}\right]=\left[\begin{array}{c}
\boldsymbol{e}_{\boldsymbol{f}}^{l} \\
\boldsymbol{e}_{\boldsymbol{f}}^{s}
\end{array}\right],
$$

with

$$
\begin{aligned}
{\left[\mathrm{E}_{\boldsymbol{f}}^{p q}\right]_{n m} } & =-\eta_{o}\left[\hat{\boldsymbol{n}} \times \boldsymbol{f}_{n}^{p}\right] \cdot \mathcal{T}_{k_{o}} \cdot \boldsymbol{f}_{m}^{q}, \\
{\left[\boldsymbol{e}_{\boldsymbol{f}}^{p}\right]_{n} } & =\boldsymbol{f}_{n}^{p} \cdot \boldsymbol{e}^{i}
\end{aligned}
$$

Note that new unknown current coefficients $\boldsymbol{b}_{\boldsymbol{f}}^{l}$ and $\boldsymbol{b}_{\boldsymbol{f}}^{s}$ are introduced in (28). These should be interpreted as in equation (19). The introduction of new coefficients is required because, 
although the EFIE describes the same physical problem as the MFIE, the solution is nonetheless different due to the discretization.

Using scaling property (10) and assuming that the diagonal blocks of the standard EFIE matrix are invertible, a Schur complement analysis shows that

$$
\left[\begin{array}{ll}
\mathrm{E}_{f}^{l l} & \mathrm{E}_{f}^{l s} \\
\mathrm{E}_{f}^{s l} & \mathrm{E}_{f}^{s s}
\end{array}\right]^{-1}=\left[\begin{array}{ll}
\mathcal{O}\left(\frac{1}{\omega}\right) & \mathcal{O}(\omega) \\
\mathcal{O}(\omega) & \mathcal{O}(\omega)
\end{array}\right]
$$

The frequency scaling of the right hand side in equation (28) can be obtained by interchanging the indices $e \leftrightarrow m$ in (25)

$$
\begin{aligned}
& {\left[\boldsymbol{e}_{\hat{\boldsymbol{n}} \times \boldsymbol{g}}^{l}\right]_{n}=\mathcal{O}\left(\omega^{\min \left(\alpha_{m}+2, \beta_{m}+1, \alpha_{e}+1\right)}\right),} \\
& {\left[\boldsymbol{e}_{\hat{\boldsymbol{n}} \times \boldsymbol{g}}^{s}\right]_{n}=\mathcal{O}\left(\omega^{\min \left(\alpha_{m}, \beta_{e}, \alpha_{e}+1\right)}\right) .}
\end{aligned}
$$

The scaling of the solution then becomes

$$
\begin{aligned}
{\left[\begin{array}{l}
\boldsymbol{b}_{\boldsymbol{f}}^{l} \\
\boldsymbol{b}_{\boldsymbol{f}}^{s}
\end{array}\right] } & =\left[\begin{array}{ll}
\mathcal{O}\left(\frac{1}{\omega}\right) & \mathcal{O}(\omega) \\
\mathcal{O}(\omega) & \mathcal{O}(\omega)
\end{array}\right] \cdot\left[\begin{array}{c}
\mathcal{O}\left(\omega^{\min \left(\alpha_{m}+2, \beta_{m}+1, \alpha_{e}+1\right)}\right) \\
\mathcal{O}\left(\omega^{\min \left(\alpha_{m}, \alpha_{e}+1, \beta_{e}\right)}\right)
\end{array}\right] \\
& =\left[\begin{array}{c}
\mathcal{O}\left(\omega^{\min \left(\alpha_{m}+1, \beta_{m}, \alpha_{e}\right)}\right) \\
\mathcal{O}\left(\omega^{\min \left(\alpha_{m}+1, \alpha_{e}+2, \beta_{e}+1\right)}\right)
\end{array}\right]
\end{aligned}
$$

which is exactly the same scaling as for the solution of the mixed MFIE. Since the standard EFIE is generally believed to yield trustworthy solutions, this correspondence between the mixed MFIE and the standard EFIE lends credibility to the correctness of the mixed MFIE solution scaling.

\section{B. Comparison with the continuous MFIE and EFIE}

The solution scaling given in (26) will now be shown to be consistent with the continuous EFIE and MFIE as well. Since no discretization is done, both these equations describe the same physical problem and lead to exactly the same solution current (barring resonances). Therefore, it is allowed to derive the frequency scaling for the nonsolenoidal and solenoidal parts of the solution current from the EFIE and MFIE respectively.

Let us start with the nonsolenoidal current. When the continuous EFIE operator acts on a nonsolenoidal current, both the scalar and vector potential part contribute. However, in the low frequency limit, the scalar potential part dominates the vector potential part. This leaves us to analyze only the dominant part which, up to some constants, is

$$
\left.\hat{\boldsymbol{n}}(\boldsymbol{r}) \times \int_{\Gamma} \nabla \nabla G_{k}\left(\boldsymbol{r}-\boldsymbol{r}^{\prime}\right)\right] \cdot \boldsymbol{j}\left(\boldsymbol{r}^{\prime}\right) \mathrm{d} S^{\prime}
$$

It turns out that, for $\omega=0$, no nonsolenoidal current can be found for which this operator yields a zero result $\forall \boldsymbol{r} \in \Gamma$. Indeed, if such a current would exist, a vector field $\Phi(\boldsymbol{r})$ could be defined as

$$
\left.\boldsymbol{\Phi}(\boldsymbol{r})=\int_{\Gamma} \nabla \nabla G_{0}\left(\boldsymbol{r}-\boldsymbol{r}^{\prime}\right)\right] \cdot \boldsymbol{j}\left(\boldsymbol{r}^{\prime}\right) \mathrm{d} S^{\prime},
$$

which has the property that $\hat{\boldsymbol{n}}(\boldsymbol{r}) \times \boldsymbol{\Phi}(\boldsymbol{r})=0, \forall \boldsymbol{r} \in \Gamma$. In addition, $\boldsymbol{\Phi}(\boldsymbol{r})$ is curl-free and divergence-free both inside and outside the scatterer (not on the boundary). Leveraging Lemma 2.2 from [37] and the fact that the scatterer is simply connected, this means that $\boldsymbol{\Phi}(\boldsymbol{r})=0$. As a consequence, the normal component of $\boldsymbol{\Phi}(\boldsymbol{r})$ does not jump when going from the inside to the outside of $\Gamma$, which means that the current is solenoidal. Since this contradicts our initial assumption, it is safe to conclude that operator (34) does not have a null-space when the frequency is zero. Therefore, if the solution current of the continuous EFIE has a nonsolenoidal component with scaling $\omega^{\nu}$, it always generates a nonzero tangential electric field with scaling $\omega^{\nu-1}$. Because the incoming electric field scales as $\mathcal{O}\left(\omega \min \left(\alpha_{m}, \beta_{e}, \alpha_{e}+1\right)\right)$, this allows the conclusion that the nonsolenoidal part of the solution current scales like $\mathcal{O}\left(\omega^{\min \left(\alpha_{m}+1, \beta_{e}+1, \alpha_{e}+2\right)}\right)$. This is the same scaling as the one obtained from the mixed MFIE and the standard EFIE.

The scaling of the solenoidal current can be deduced from the continuous MFIE. Indeed, from the fact that the continuous MFIE operator has a DC limit, it immediately follows that the complete solution current has the same frequency scaling as the incoming tangential magnetic field if the MFIE does not have a null-space at $\omega=0$. Since the scatterer is simply connected, such a null-space never occurs [37], which explains the $\mathcal{O}\left(\omega^{\min \left(\alpha_{m}+1, \beta_{m}, \alpha_{e}\right)}\right)$ scaling of the solenoidal current coefficients in (26). Of course, the nonsolenoidal current coefficients should also obey this scaling law, since it was derived for the complete current, not only for the solenoidal part. It can be quickly checked that this is indeed the case because

$$
\min \left(\alpha_{m}+1, \alpha_{e}+2, \beta_{e}+1\right) \geq \min \left(\alpha_{m}+1, \beta_{m}, \alpha_{e}\right),
$$

always holds.

\section{LF BEHAVIOR OF THE MUIE}

The MUIE is an integral equation of the second kind that is applicable when $\Omega$ is filled with a dielectric material (permittivity $\varepsilon_{i}$, permeability $\mu_{i}$, wave number $k_{i}=\omega \sqrt{\varepsilon_{i} \mu_{i}}$ ). The MUIE uses both an electric and a magnetic current distribution

$$
\begin{array}{r}
{\left[\begin{array}{cc}
\varepsilon_{o} \mathcal{K}_{k_{o}}-\varepsilon_{i} \mathcal{K}_{k_{i}}-\varepsilon_{m} \mathcal{I} & \frac{k_{i}}{\omega} \mathcal{T}_{k_{i}}-\frac{k_{o}}{\omega} \mathcal{T}_{k_{o}} \\
\frac{k_{o}}{\omega} \mathcal{T}_{k_{o}}-\frac{k_{i}}{\omega} \mathcal{T}_{k_{i}} & \mu_{o} \mathcal{K}_{k_{o}}-\mu_{i} \mathcal{K}_{k_{i}}-\mu_{m} \mathcal{I}
\end{array}\right] \cdot\left[\begin{array}{c}
\boldsymbol{m} \\
\boldsymbol{j}
\end{array}\right]} \\
=\left[\begin{array}{c}
\varepsilon_{o} \hat{\boldsymbol{n}} \times \boldsymbol{e}^{i} \\
-\mu_{o} \hat{\boldsymbol{n}} \times \boldsymbol{h}^{i}
\end{array}\right],
\end{array}
$$

where $\varepsilon_{m}=\frac{\varepsilon_{o}+\varepsilon_{i}}{2}, \mu_{m}=\frac{\mu_{o}+\mu_{i}}{2}$. Compared to the MFIE, the new thing here is the appearance of the $\mathcal{T}$ operators. When the MUIE is discretized using the mixed scheme, equations (8), (9) and (11) can be used to determine the frequency scaling of the various blocks:

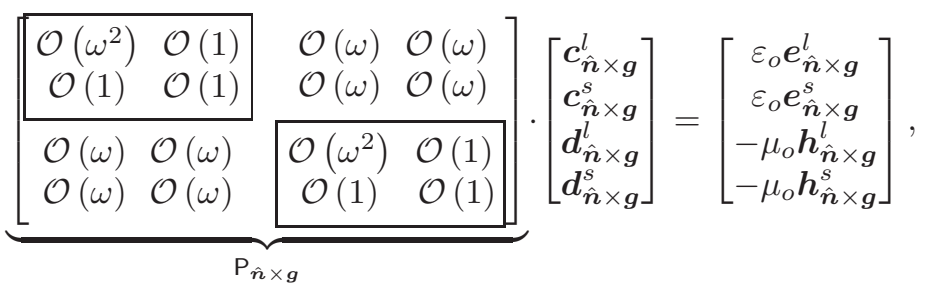

where the coefficients $c_{t}$ and $\boldsymbol{d}_{t}$ represent the magnetic and electric current respectively. It is immediately seen that the 
sub-blocks of the two framed blocks have the same frequency scaling as the sub-blocks of the mixed MFIE, as given in equation (23). In addition, they have the same sizes. Therefore, assuming that the two framed blocks are invertible at zero frequency, equation (24) yields the frequency scalings of the sub-blocks of their inverses. A Schur complement reasoning then yields

$$
\mathrm{P}_{\hat{\boldsymbol{n}} \times \boldsymbol{g}}^{-1}=\left[\begin{array}{cccc}
\mathcal{O}(1) & \mathcal{O}(1) & \mathcal{O}(\omega) & \mathcal{O}(\omega) \\
\mathcal{O}(1) & \mathcal{O}\left(\omega^{2}\right) & \mathcal{O}(\omega) & \mathcal{O}(\omega) \\
\mathcal{O}(\omega) & \mathcal{O}(\omega) & \mathcal{O}(1) & \mathcal{O}(1) \\
\mathcal{O}(\omega) & \mathcal{O}(\omega) & \mathcal{O}(1) & \mathcal{O}\left(\omega^{2}\right)
\end{array}\right]
$$

Using the frequency scalings of the incoming fields from equations (25) and (32), the frequency scaling of the electric and magnetic current coefficients can be shown to be

$$
\left[\begin{array}{c}
\boldsymbol{c}_{\hat{\boldsymbol{n}} \times \boldsymbol{g}}^{l} \\
\boldsymbol{c}_{\hat{n} \times \boldsymbol{g}}^{s} \\
\boldsymbol{d}_{\hat{\boldsymbol{n}} \times \boldsymbol{g}}^{l} \\
\boldsymbol{d}_{\hat{\boldsymbol{n}} \times \boldsymbol{g}}^{s}
\end{array}\right]=\left[\begin{array}{c}
\mathcal{O}\left(\omega^{\min \left(\alpha_{e}+1, \alpha_{m}, \beta_{e}\right)}\right) \\
\mathcal{O}\left(\omega^{\min \left(\beta_{m}+1, \alpha_{e}+1, \alpha_{m}+2\right)}\right) \\
\mathcal{O}\left(\omega^{\min \left(\alpha_{m}+1, \alpha_{e}, \beta_{m}\right)}\right) \\
\mathcal{O}\left(\omega^{\min \left(\beta_{e}+1, \alpha_{m}+1, \alpha_{e}+2\right)}\right)
\end{array}\right]
$$

It can be seen that the frequency scaling of the electric current is the same as that of the electric current obtained from PEC scattering. The scaling of the magnetic current is obtained from that of the electric current by interchanging the indices $e \leftrightarrow m$, which is consistent with the duality between electric and magnetic fields.

Similarly to what was done in Subsection (V-A), the frequency scaling of the currents can also be obtained from the standard PMIE, which yields exactly the same result as in (40). In contrast, the standard discretization of the MUIE yields

$$
\left[\begin{array}{l}
\boldsymbol{c}_{\hat{\boldsymbol{n}} \times \boldsymbol{g}}^{l} \\
\boldsymbol{c}_{\hat{n} \times \boldsymbol{g}}^{\mathrm{s}} \\
\boldsymbol{d}_{\hat{\boldsymbol{n}} \times \boldsymbol{g}}^{l} \\
\boldsymbol{d}_{\hat{\boldsymbol{n}} \times \boldsymbol{g}}^{s}
\end{array}\right]=\left[\begin{array}{c}
\mathcal{O}\left(\omega^{\min \left(\alpha_{e}+1, \alpha_{m}, \beta_{e}\right)}\right) \\
\mathcal{O}\left(\omega^{\min \left(\alpha_{e}+1, \alpha_{m}, \beta_{e}\right)}\right) \\
\mathcal{O}\left(\omega^{\min \left(\alpha_{m}+1, \alpha_{e}, \beta_{m}\right)}\right) \\
\mathcal{O}\left(\omega^{\min \left(\alpha_{m}+1, \alpha_{e}, \beta_{m}\right)}\right)
\end{array}\right],
$$

which is again not physical for certain excitations.

\section{NUMERICAL RESUlTS}

To corroborate the theoretical results from the earlier sections, numerical tests will be performed for both a PEC and dielectric scatterer. The test will be limited to one geometry to keep the length of this section within acceptable bounds. The generality of the numerical results is ensured by choosing a geometry that contains smooth curved surfaces as well as a sharp edge and a corner. Figure 1 shows a mesh, containing 990 edges, of the geometry that was chosen.

Four types of excitations are used: an electric loop current (EO), an electric line current (EI), a magnetic loop current (MO) and a magnetic line current (MI). These currents are unit currents that run over an infinitesimally thin wire that is either loop- or line-shaped. The loop is located in the $x z$-plane, has center $[2,0,1] \mathrm{m}$ and radius of $0.5 \mathrm{~m}$. The line is straight and connects the point $\left[2,0, \frac{1}{2}\right] \mathrm{m}$ with $\left[2,0, \frac{3}{2}\right] \mathrm{m}$. Figure 1 graphically depicts the positions of the loop and line. The

\begin{tabular}{|c|c|c|c|c|}
\hline Excitation & $\alpha_{e}$ & $\beta_{e}$ & $\alpha_{m}$ & $\beta_{m}$ \\
\hline \hline EO & 0 & $\infty$ & $\infty$ & $\infty$ \\
\hline EI & 0 & -1 & $\infty$ & $\infty$ \\
\hline MO & $\infty$ & $\infty$ & 0 & $\infty$ \\
\hline MI & $\infty$ & $\infty$ & 0 & -1 \\
\hline
\end{tabular}

TABLE I

THE FREQUENCY SCALINGS OF THE FOUR TESTED EXCITATIONS.

frequency scalings for these four excitations are easily derived and given in Table I.
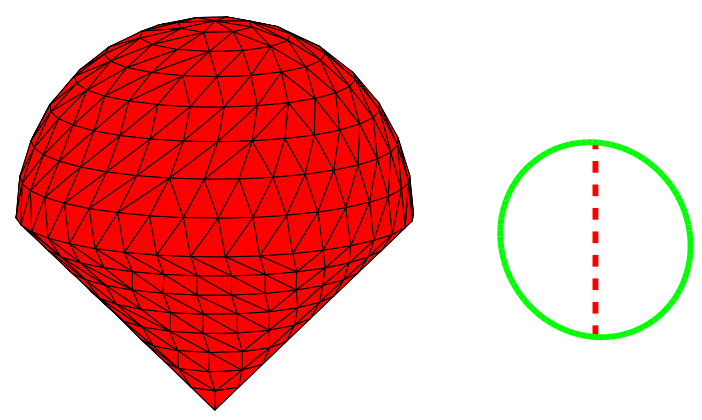

Fig. 1. The mesh (990 edges) used for discretizing the scatterer, along with the loop (green) and line (dashed red) excitation currents. The scatterer consists of a hemisphere (with radius $1 \mathrm{~m}$ and center $[0,0,1] \mathrm{m}$ ) and a cone with its tip located at the origin. The positioning of the scatterer and sources in this figure is the same as in the simulations.

For each of these four excitations, three different solution currents are computed. All three are linear combinations of the RWGs defined on the mesh in Figure 1. The first solution current is obtained by means of the standard EFIE or PMIE (for a PEC or dielectric scatterer respectively). The second solution current is obtained from the mixed MFIE or the mixed MUIE, while the third solution current is obtained from the standard MFIE or standard MUIE. For each solution current, we will focus solely on the electric part of the solution current. Neglecting the magnetic solution current is not a loss of generality because it is zero for PECs. For the dielectric case, the frequency scaling of the magnetic current can be obtained from the electric current scaling by means of duality. Therefore, it is not necessary to further consider the magnetic current.

Finally, the electric part of the solution current is split into a solenoidal part $j^{s}$ and a nonsolenoidal part $j^{n}$, which is taken to be the $L_{2}$-orthogonal complement of the solenoidal part. The frequency scalings derived in this paper immediately lead to the expected frequency scalings for both of these two parts. These are listed in Tables II and III. It will now be checked whether the scalings from II and III are reproduced numerically. First, the PEC scatterer case will be considered, using the mesh shown in Figure 1. Figures 3(a), 3(b), 3(c) and 3(d) show the numerically obtained $L_{2}$ norm of the solenoidal and nonsolenoidal parts of the solution current. For low frequencies, an excellent agreement between the theoretically predicted and numerically obtained frequency scalings is observed. For an electric line and magnetic loop excitation, all three solution currents exhibit the same frequency scaling. However, for an electric loop or magnetic line current, the 
nonsolenoidal part of the standard MFIE solution current is wrong at sufficiently low frequencies.

It should be noted that, if the frequency is lowered further and further, the standard EFIE and mixed MFIE will eventually yield wrong solution currents as well. However, this is purely due to the numerical rounding error inherent in floating-point arithmetic, not due to the discretization scheme. For example, if one were to switch to a high-precision data type, this problem could in principle be solved for arbitrarily low frequencies. For the standard MFIE, however, the wrong current scaling is inherent in the discretization scheme and cannot be remedied by performing more accurate computations. It can be remedied by changing the equation, though, as is done for example in [20].

For the case of a penetrable scatterer, the permittivity and permeability were chosen to be $\varepsilon=3 \varepsilon_{0}$ and $\mu=\frac{4}{3} \mu_{0}$ respectively. Again, the mesh in Figure 1 was used. Figures 4(a), 4(b), 4(c) and 4(d) show the numerically obtained scalings. Again, all the predicted frequency scalings are confirmed. In figures 4(a) and 4(d), the PMIE departs from the expected scaling when $k$ becomes smaller than approximately $10^{-5}$. This effect is caused by the numerical rounding error mentioned above.

With a final numerical result, it will be shown that the frequency scalings obtained here are also valid for other mesh densities. Figure 5 shows the $L_{2}$ norm of the nonsolenoidal part of the solution current obtained from the mixed and standard MUIE, discretized with 63, 252 and 990 edges. The latter mesh is shown in Figure 1 and the two coarser meshes are shown in Figure 2. It can be seen in Figure 5 that the frequency scaling for the mixed discretization remains correct, irrespective of how coarse the mesh is. For the standard discretization, however, the plots indicate that a coarser discretization leads to an earlier incorrect frequency scaling of the nonsolenoidal current. In this context, 'earlier' means that the onset of the problem occurs at higher frequencies. This is consistent with the knowledge that the standard MFIE or MUIE converge to the correct solution in the $L_{2}$ norm (this convergence is widely known from experience but can also be proved, similarly to the proof given for the mixed MFIE in [17]). Indeed, if one keeps on refining the mesh but keeps the frequency constant, the correct solution will eventually be obtained. However, a lower frequency directly translates into a requirement for a denser mesh, before obtaining the correct solution. With the mixed discretization scheme, no such requirement on the mesh density exists.

\section{CONCLUSION}

A rigorous analysis of the standard and mixed discretizations of second kind integral equations is presented in the context of low-frequency scattering problems. This analysis shows that the mixed discretization of second kind integral equations yields the same solution current frequency scalings as the standard discretization of integral equations of the first kind. In addition, these frequency scalings are consistent with those obtained from the continuous equations. In contrast to this, the standard discretization of second kind integral equations does

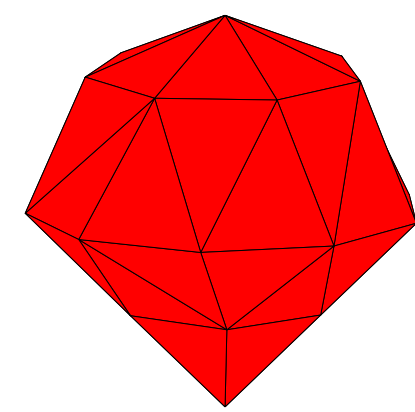

(a)

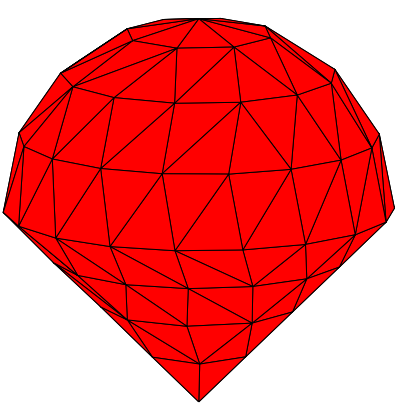

(b)
Fig. 2. The two coarser meshes used for discretizing the scatterer. Mesh 2(a) contains 63 edges and mesh 2(b) contains 252 edges.

\begin{tabular}{|c|c|c|}
\hline Excitation & Solenoidal & Nonsolenoidal \\
\hline \hline EO & $\mathcal{O}(1)$ & $\mathcal{O}\left(\omega^{2}\right)$ \\
\hline EI & $\mathcal{O}(1)$ & $\mathcal{O}(1)$ \\
\hline MO & $\mathcal{O}(\omega)$ & $\mathcal{O}(\omega)$ \\
\hline MI & $\mathcal{O}\left(\omega^{-1}\right)$ & $\mathcal{O}(\omega)$ \\
\hline
\end{tabular}

TABLE II

THE THEORETICALLY PREDICTED FREQUENCY SCALINGS OF THE SOLENOIDAL AND NONSOLENOIDAL PART OF THE ELECTRIC SOLUTION CURRENT WHEN EITHER A STANDARD DISCRETIZATION OF AN INTEGRAL EQUATION OF THE FIRST KIND IS USED, OR A MIXED DISCRETIZATION OF AN INTEGRAL EQUATION OF THE SECOND KIND IS USED.

not yield the correct frequency scalings for the nonsolenoidal part of the solution current whenever, broadly speaking, the excitation was generated by a divergence-less electric current or a magnetic current with nonzero divergence. Numerical results back up these theoretical predictions. The incorrect frequency scaling of the standard discretization implies that, when the frequency is lowered, the standard discretization requires a finer and finer mesh to attain a constant accuracy. The mixed discretization does not suffer from this problem. Therefore, at low frequencies, it can be more efficient than the standard discretization, despite the considerable complexity of the $\mathrm{BC}$ or $\mathrm{CW}$ test functions.

\begin{tabular}{|c|c|c|}
\hline Excitation & Solenoidal & Nonsolenoidal \\
\hline \hline EO & $\mathcal{O}(1)$ & $\mathcal{O}(1)$ \\
\hline EI & $\mathcal{O}(1)$ & $\mathcal{O}(1)$ \\
\hline MO & $\mathcal{O}(\omega)$ & $\mathcal{O}(\omega)$ \\
\hline MI & $\mathcal{O}\left(\omega^{-1}\right)$ & $\mathcal{O}\left(\omega^{-1}\right)$ \\
\hline
\end{tabular}

TABLE III

THE THEORETICALLY PREDICTED FREQUENCY SCALINGS OF THE SOLENOIDAL AND NONSOLENOIDAL PART OF THE ELECTRIC SOLUTION CURRENT WHEN A STANDARD DISCRETIZATION OF AN INTEGRAL EQUATION OF THE SECOND KIND IS USED. 


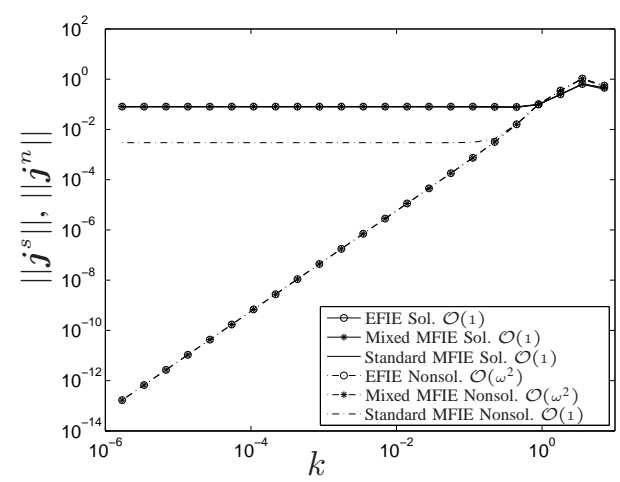

(a)

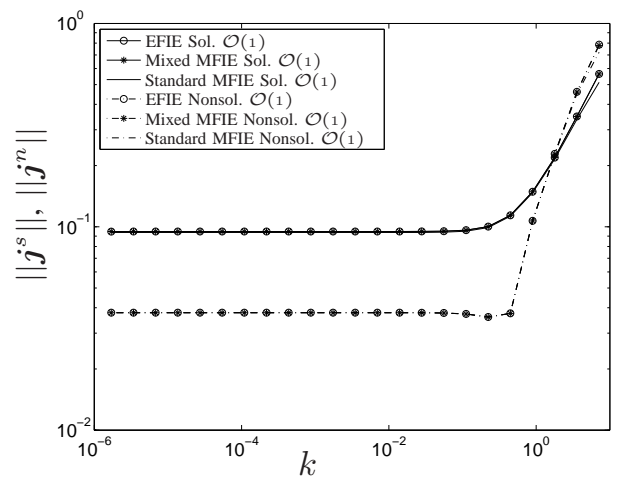

(b)

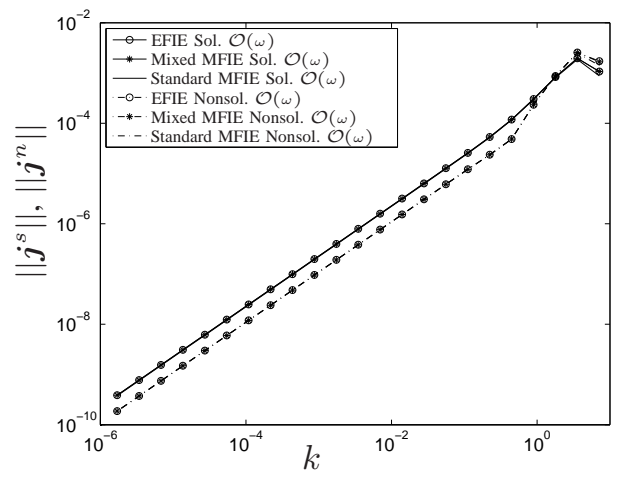

(c)

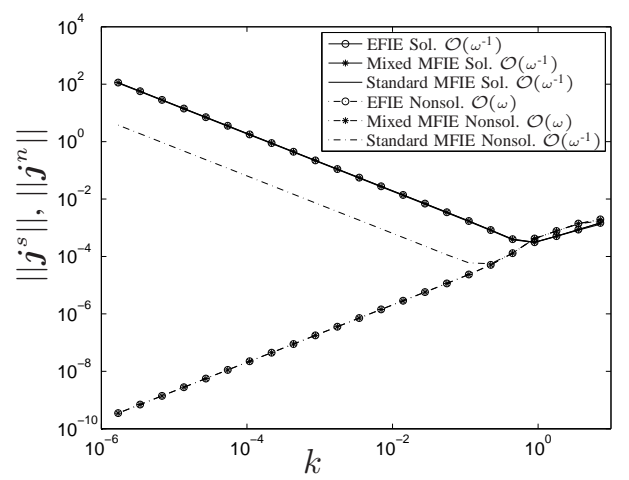

(d)

Fig. 3. The frequency scalings of the solenoidal (Sol.) and nonsolenoidal (Nonsol.) part of the electric solution current, obtained for scattering by a PEC object for four excitations: an electric loop current (a), an electric line current (b), a magnetic loop current (c) and a magnetic line current (d). The scalings in the legends are the theoretically expected ones.

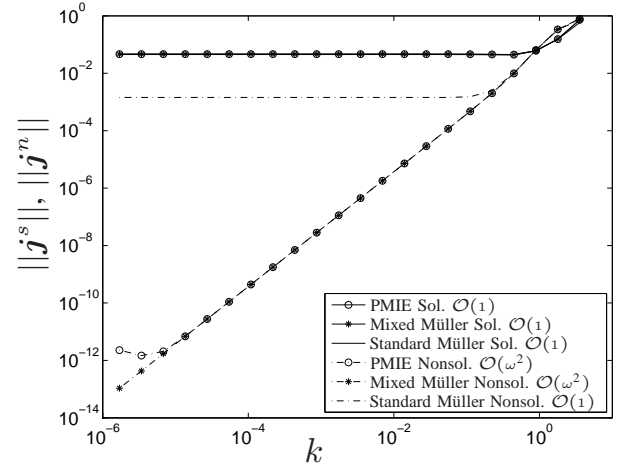

(a)

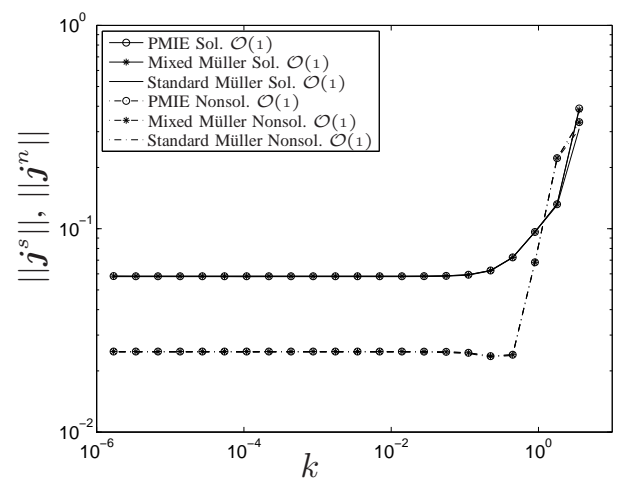

(b)

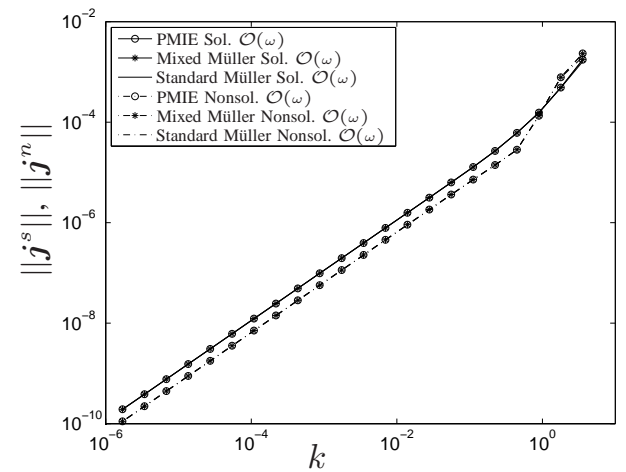

(c)

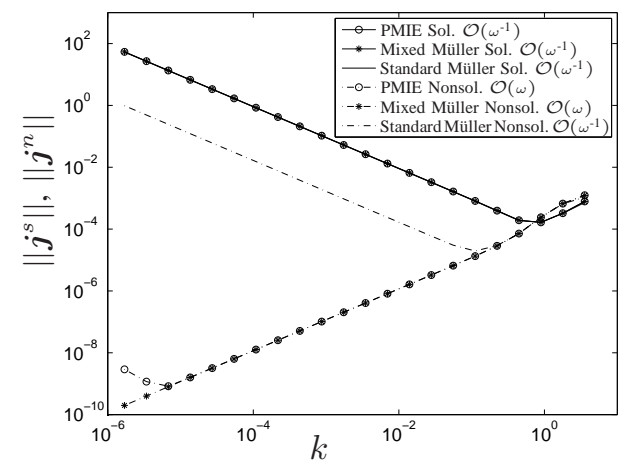

(d)

Fig. 4. The frequency scalings of the solenoidal (Sol.) and nonsolenoidal (Nonsol.) part of the electric solution current, obtained for scattering by a penetrable object $\left(\varepsilon=3 \varepsilon_{0}, \mu=\frac{4}{3} \mu_{0}\right)$ for four excitations: an electric loop current (a), an electric line current (b), a magnetic loop current (c) and a magnetic line current (d). The scalings in the legends are the theoretically expected ones. 


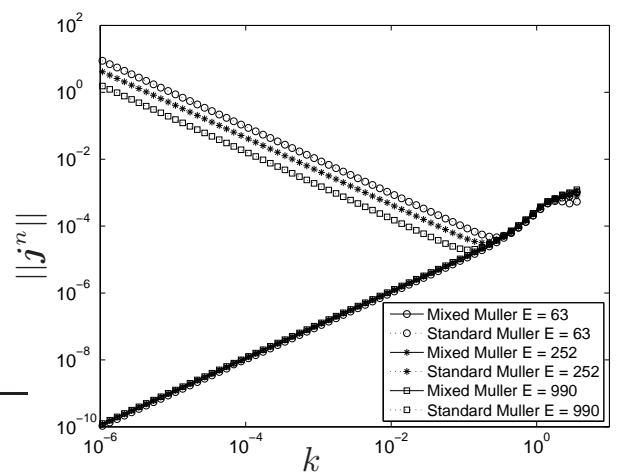

Fig. 5. The frequency scaling of the nonsolenoidal part of the solution current for scattering by a penetrable object (the same as for Figure 4), with a magnetic line current as excitation, obtained with three different meshes. The number of edges $E$ in the meshes is 63,252 and 990 . It can be seen that the mixed discretization yields the correct frequency scaling for all frequencies, whereas the standard discretization requires a finer and finer mesh to get an accurate nonsolenoidal part as the frequency is lowered.

\section{APPENDIX A}

Some details of the Schur complement analysis will be discussed here. Assume that $Q$ is a 2 by 2 block matrix

$$
\mathrm{Q}=\left[\begin{array}{ll}
\mathrm{Q}_{11} & \mathrm{Q}_{12} \\
\mathrm{Q}_{21} & \mathrm{Q}_{22}
\end{array}\right] \text {, }
$$

with square and invertible off-diagonal blocks. This corresponds to the mixed MFIE case (23). The blocks of $Q^{-1}$ can be written as follows

$$
Q^{-1}=\left[\begin{array}{cc}
-P_{1} \cdot Q_{22} \cdot Q_{12}^{-1} & P_{1} \\
P_{2} & -P_{2} \cdot Q_{11} \cdot Q_{21}^{-1}
\end{array}\right],
$$

with

$$
\begin{aligned}
& P_{1}=\left[Q_{21}-Q_{22} \cdot Q_{12}^{-1} \cdot Q_{11}\right]^{-1}, \\
& P_{2}=\left[Q_{12}-Q_{11} \cdot Q_{21}^{-1} \cdot Q_{22}\right]^{-1} .
\end{aligned}
$$

The inverses of $P_{1}$ and $P_{2}$ are the Schur complements of $Q_{12}$ and $Q_{21}$ respectively [36]. The scalings in (23) imply $\mathrm{P}_{1}=\mathcal{O}(1)$ and $\mathrm{P}_{2}=\mathcal{O}(1)$, such that the scalings in (24) are immediately obtained.

For the case of the standard EFIE, the diagonal blocks of $Q$ are square and invertible. This leads to a modified formula for the inverse:

$$
\mathrm{Q}^{-1}=\left[\begin{array}{cc}
\mathrm{L}_{1} & -\mathrm{L}_{1} \cdot \mathrm{Q}_{12} \cdot \mathrm{Q}_{22}^{-1} \\
-\mathrm{L}_{2} \cdot \mathrm{Q}_{21} \cdot \mathrm{Q}_{11}^{-1} & \mathrm{~L}_{2}
\end{array}\right],
$$

with

$$
\begin{aligned}
& \mathrm{L}_{1}=\left[\mathrm{Q}_{11}-\mathrm{Q}_{12} \cdot \mathrm{Q}_{22}^{-1} \cdot \mathrm{Q}_{21}\right]^{-1}, \\
& \mathrm{~L}_{2}=\left[\mathrm{Q}_{22}-\mathrm{Q}_{21} \cdot \mathrm{Q}_{11}^{-1} \cdot \mathrm{Q}_{12}\right]^{-1} .
\end{aligned}
$$

This expression leads to the scalings in equation (31).

For the case of the 4 by 4 block matrix arising in the MUIE, it is advantageous to proceed in two steps. First, treat the matrix in (38) as the 2 by 2 block matrix

$$
\left[\begin{array}{cc}
\text { A } & \mathcal{O}(\omega) \\
\mathcal{O}(\omega) & \text { B }
\end{array}\right]
$$

Here, the two diagonal blocks are square and invertible. Therefore, equation (46) can be used. However, the matrices $L_{1}$ and $L_{2}$ depend on the inverses of $A$ and $B$. At that point it becomes necessary to take into account the sub-blocks of these matrices. Since A and B have square and invertible offdiagonal blocks, equation (43) can be used to show that their inverses have scaling

$$
A^{-1}=\left[\begin{array}{cc}
\mathcal{O}(1) & \mathcal{O}(1) \\
\mathcal{O}(1) & \mathcal{O}\left(\omega^{2}\right)
\end{array}\right]=\mathrm{B}^{-1} .
$$

This implies that

$$
\mathrm{L}_{1}=\left[\begin{array}{cc}
\mathcal{O}(1) & \mathcal{O}(1) \\
\mathcal{O}(1) & \mathcal{O}\left(\omega^{2}\right)
\end{array}\right]=\mathrm{L}_{2},
$$

The scaling result in equation (39) immediately follows from this and (46).

\section{ACKNOWLEDGMENT}

Ignace Bogaert is supported by a postdoctoral grant from the Fund for Scientific Research Flanders (FWO-Vlaanderen).

\section{REFERENCES}

[1] Poggio, A. and Miller, E., "Integral Equation Solutions of Threedimensional Scattering Problems," Computer Techniques for Electromagnetics, R. Mittra, ed. (Pergamon, New York, 1973).

[2] S. D. Rao, D. R. Wilton, and A. W. Glisson, "Electromagnetic Scattering by Surfaces of Arbitrary Shape," IEEE Trans. Antennas Propag., vol. AP-30, no. 3, May 1982.

[3] F. Murray, "Conductors in an Electromagnetic Field," Ant. J. Math., vol. 53, pp. 275-288, 1931.

[4] A. Maue, "On the Formulation of a General Scattering Problem by Means of an Integral Equation," Z. Phys., vol. 126(7/9), pp. 601-618, 1949.

[5] Müller, C., Foundations of the Theory of Electromagnetic Waves. Springer Verlag, 1969.

[6] E. Úbeda and J.M. Rius, "MFIE MoM-Formulation with CurlConforming Basis Functions and Accurate Kernel-Integration in the Analysis of Perfectly Conduction Sharp-Edged Objects," Microwave and Optical Technology Letters, vol. 44, no. 4, pp. 354-358, Feb 2005.

[7] F. Andriulli, K. Cools, H. Bagci, F. Olyslager, A. Buffa, S. Christiansen, and E. Michielssen, "A Multiplicative Calderon Preconditioner for the Electric Field Integral Equation," IEEE Transactions on Antennas and Propagation, vol. 56, no. 8, pp. 2398-2412, Aug 2008.

[8] L. Greengard and V. Rokhlin, "A Fast Algorithm for Particle Simulations," Journal of Computational Physics, vol. 73, no. 2, pp. 325-348, Dec. 1987.

[9] J. Song, C.C. Lu, and W.C. Chew, "Multilevel Fast Multipole Algorithm for Electromagnetic Scattering by Large Complex Objects," IEEE Transactions on Antennas and Propagation, vol. 45, no. 10, pp. 1488-1493, Oct. 1997.

[10] W.C. Chew, J.M. Jin, C.C. Lu, E. Michielssen, and J.M. Song, "Fast Solution Methods in Electromagnetics," IEEE Transactions on Antennas and Propagation, vol. 45, no. 3, pp. 533-543, Mar. 1997.

[11] E. Úbeda, A. Heldring and J.M. Rius, "Accurate Computation of the Impedance Elements of the Magnetic-Field Integral Equation with RWG Basis Functions Through Field-Domain and Source-Domain Integral Swapping," Microwave and Optical Technology Letters, vol. 49, no. 3, pp. 709-712, March 2007. 
[12] L. Zhang, A. Deng, and M. Wang, "Improving the Accuracy of the Magnetic Field Integral Equation with the Use of a new Impedance Matrix Element Formulation," IET Microwaves, Antennas and Propagation, vol. 3, no. 5, pp. 850-855, 2009.

[13] Ö. Ergül and L. Gürel, "Solid-Angle Factor in the Magnetic-Field Integral Equation," Microwave and Optical Technology Letters, vol. 45, no. 5, pp. 452-456, June 2005.

[14] E. Úbeda and J.M. Rius, "Novel Monopolar MFIE MoM-Discretization for the Scattering Analysis of Small Objects," IEEE Transactions on Antennas and Propagation, vol. 54, no. 1, pp. 50-57, Jan 2006

[15] Ö. Ergül and L. Gürel, "Linear-Linear Basis Functions for MLFMA Solutions of Magnetic-Field and Combined-Field Integral Equations," IEEE Transactions on Antennas and Propagation, vol. 55, no. 4, pp. 1103-1110, Apr 2007.

[16] K. Cools, F. Andriulli, F. Olyslager, and E. Michielssen, "Improving the MFIE's Accuracy by Using a Mixed Discretization," in Antennas and Propagation Society International Symposium, June 2009, pp. 1-4.

[17] K. Cools, F. Andriulli, D. De Zutter, and E. Michielssen, "Accurate and Conforming Mixed Discretization of the MFIE," IEEE Antennas and Wireless Propagation Letters, vol. 10, pp. 528-531, 2011.

[18] A. Buffa and S. H. Christiansen, "A Dual Finite ELement Complex on the Barycentric Refinement," Math. of Comp., vol. 260, pp. 1743-1769, 2007.

[19] Q. Chen and D. Wilton, "Electromagnetic Scattering by Threedimensional Arbitrary Complex Material/Conducting Bodies," Proceedings of the IEEE Symposium on Antennas and Propagation, vol. 2, pp. 590-593, 1990.

[20] Y. Zhang, C. T.J., W. Chew, and J. Zhao, "Magnetic Field Integral Equation at Very Low Frequencies," IEEE Transactions on Antennas and Propagation, vol. 51, no. 8, pp. 1864-1871, Aug 2003.

[21] Reddy, J.N., An Introduction to the Finite Element Method. Mc Graw Hill, 2005

[22] F. Vico, Z. Gimbutas, L. Greengard, and M. Ferrando-Bataller, "Overcoming Low-Frequency Breakdown of the Magnetic Field Integral Equation," Antennas and Propagation, IEEE Transactions on, vol. 61, no. 3, pp. 1285-1290, 2013.

[23] E. Ubeda, J. Tamayo, J. Rius, and A. Heldring, "Stable Discretization of the Electric-Magnetic Field Integral Equation With the TaylorOrthogonal Basis Functions," Antennas and Propagation, IEEE Transactions on, vol. 61, no. 3, pp. 1484-1490, 2013.

[24] I. Bogaert, K. Cools, F. P. Andriulli, J. Peeters, and D. De Zutter, "Low Frequency Stability of the Mixed Discretization of the MFIE," in Proceedings of the 5th European Conference on Antennas and Propagation, Rome, Italy, 11-15 April 2011.

[25] I. Bogaert, K. Cools, F. Andriulli, and D. De Zutter, "Low Frequency Scaling of the Mixed MFIE for Scatterers with a Non-Simply Connected Surface," in Proceedings of the International Conference on Electromagnetics in Advanced Applications, Torino, Italy, 12-17 September 2011.

[26] K. Cools, F. Andriulli, I. Bogaert, and D. De Zutter, "The Magnetic Field Integral Equation: Spatial and Frequency Behavior," in Proceedings of the Workshop on Advanced Techniques in Computational Electromagnetics (AT-CEM 2011), Glasgow, United Kingdom, 20-22 June 2011.

[27] S. P. Kiminki, I. Bogaert, and P. Yl-Oijala, "Dual Basis for the Fully Linear LL Functions," in Proceedings of the IEEE International Symposium on Antennas and Propagation and USNC-URSI National Radio Science Meeting, Chicago, Illinois, 8-14 July 2012.

[28] Ülkü, H. A. and Bogaert, I. and Cools, K. and Andriulli, F. P. and Bagci, H., "On the Low Frequency Behavior of the Mixed Discretized Time Domain Magnetic Field Integral Equation," in Proceedings of the IEEE International Symposium on Antennas and Propagation and USNC-URSI National Radio Science Meeting, Chicago, Illinois, 8-14 July 2012.

[29] - "On the Mixed Discretization of the Time Domain Magnetic Field Integral Equation," in Proceedings of the International Conference on Electromagnetics in Advanced Applications, Cape Town, South Africa, 2-7 September 2012.

[30] S. Chen, W. Chew, J. Song, and J.-S. Zhao, "Analysis of Low Frequency Scattering from Penetrable Scatterers," IEEE Transactions on Geoscience and Remote Sensing, vol. 4, no. 39, p. 726, 7352001.

[31] K. Cools, F. Andriulli, F. Olyslager, and E. Michielssen, "Time Domain Calderón Identities and Their Application to the Integral Equation Analysis of Scattering by PEC Objects Part I: Preconditioning," IEEE Transactions on Antennas and Propagation, vol. 57, no. 8, pp. 23522364, Aug 2009.

[32] G. Vecchi, "Loop-Star Decomposition of Basis Functions in the Discretization of the EFIE," IEEE Transactions on Antennas and Propagation, vol. 47, no. 2, pp. 339-346, Feb 1999.
[33] P. Yla-Oijala and M. Taskinen, "Well-Conditioned Muller Formulation for Electromagnetic Scattering by Dielectric Objects," IEEE Transactions on Antennas and Propagation, vol. 53, no. 10, pp. 3316-3323, 2005.

[34] J.-S. Zhao and W. C. Chew, "Integral Equation Solution of Maxwell's Equations from Zero Frequency to Microwave Frequencies," IEEE Transactions on Antennas and Propagation, vol. 48, pp. 1635-1645, Oct. 2000

[35] M. Stephanson and J. F. Lee, "Preconditioned Electric Field Integral Equation using Calderon Identities and Dual Loop/Star Basis Functions," IEEE Transactions on Antennas and Propagation, vol. 57, no. 4, pp. 1274-1279, 2009.

[36] R. W. Cottle, "Manifestations of the Schur Complement," Linear Algebra and its Applications, vol. 8, no. 3, pp. 189 - 211, 1974.

[37] K. Cools and F. P. Andriulli and F. Olyslager and E. Michielssen, "Nullspaces of MFIE and Calderon Preconditioned EFIE Operators Applied to Toroidal Surfaces," IEEE Transactions on Antennas and Propagation, vol. 57, no. 10, pp. 3205-3215, Oct 2009.

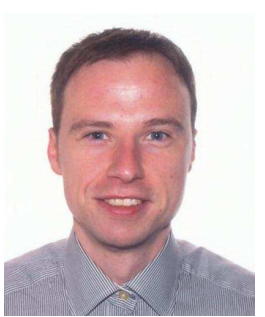

Ignace Bogaert Ignace Bogaert was born in Ghent, Belgium, in 1981. He received the M.S. degree in physics engineering from Ghent University, Ghent, Belgium, in 2004. After graduating, he joined the Electromagnetics Group of the Department of Information Technology (INTEC) at Ghent University, where he received his Ph.D in applied physics in 2008. His research is supported by a postdoctoral grant from the Research Foundation-Flanders (FWO-Vlaanderen). Dr. Bogaert was the recipient of a special young scientist recognition at ICEAAIEEE APWC, 2011. He co-authored the second prize-paper in the best paper competition of URSI-EMTS 2013. Also, he received an honorable mention at URSI/IEEE-APS 2008 and authored two other finalist conference papers (ICEAA-IEEE APWC, 2012 \& 2013). His research interests include optimization problems and the modeling of various physical systems, with the emphasis on robustness, efficiency and accuracy.

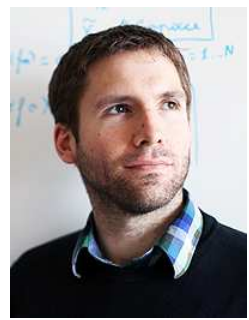

Kristof Cools Kristof Cools received the MEng degree in Applied Physics Engineering from Ghen University, Belgium, in 2004. His master's dissertation dealt with the full wave simulation of metamaterials using the low frequency multilevel fast multipole method. He received the Ph.D. degree from Ghent University in 2008, under the advisership of Prof. F. Olyslager and Prof. Eric Michielssen. In 2008, he was awarded the Young Scientist Best Paper award at the International Conference on Electromagnetics and Advanced Applications. In 2011, he was a visiting research fellow at TELECOM Bretagne. In November 2011, he took up a position as Lecturer in Computational Electromagnetics at the University of Nottingham. His current focuses on the spectral properties of the boundary integral operators of electromagnetics, on stable and accurate discretization schemes for frequency and time domain boundary element methods, on domain decomposition techniques, and on the implementations of algorithms from computational physics for high performance computing. 


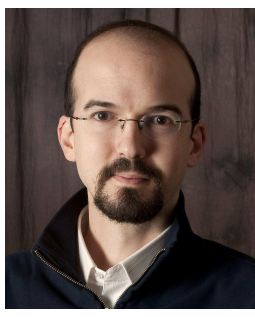

Francesco Andriulli Francesco P. Andriulli (S '05, M '09, SM '11) received the Laurea in electrical engineering from the Politecnico di Torino, Italy, in 2004, the M.Ss. in electrical engineering and computer science from the University of Illinois at Chicago in 2004, and the Ph.D. in electrical engineering from the University of Michigan at Ann Arbor in 2008. From 2008 to 2010 he was a Research Associate with the Politecnico di Torino. Since 2010 he has been with the Microwave Department of the cole nationale suprieure des tlcommunications de Bretagne (TELECOM Bretagne), Brest, France, where he currently is a Matre de confrences. His research interests are in computational electromagnetics with focus on preconditioning and fast solution of frequency and time domain integral equations, integral equation theory, hierarchical techniques, and single source integral equations. Dr. Andriulli was awarded the University of Michigan International Student Fellowship and the University of Michigan Horace H. Rackham Predoctoral Fellowship. He was the recipient of the best student paper award at the 2007 URSI North American Radio Science Meeting. He received the first place prize of the student paper context of the 2008 IEEE Antennas and Propagation Society International Symposium. He was the recipient of the 2009 RMTG Award for junior researchers and was awarded two URSI Young Scientist Awards at the International Symposium on Electromagnetic Theory in 2010 and 2013 where he was also awarded the second prize in the best paper contest. In addition Dr. Andriulli coauthored another first prize conference paper (ICEAA 2009), two honourable mention conference papers (ICEAA 2011, URSI/IEEE-APS 2013) and other three finalist conference papers (URSI/IEEE-APS 2012, URSI/IEEE-APS 2007, URSI/IEEE-APS 2006). He serves as Associate Editor for the IEEE TRANSACTIONS ON ANTENNAS AND PROPAGATION and for the IEEE ANTENNAS AND WIRELESS PROPAGATION LETTERS.

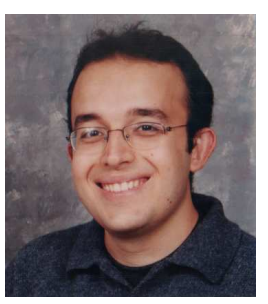

Hakan Bağcı Hakan Bağcı (S'98-M’07) received the B.S. degree in Electrical and Electronics Engineering from the Bilkent University, Ankara, Turkey, in June 2001 and the M.S. and Ph.D. degrees in Electrical and Computer Engineering from the University of Illinois at Urbana-Champaign (UIUC), Urbana, in August 2003 and January 2007, respectively. From June 1999 to July 2001, he worked as an Undergraduate Researcher at the Computational Electromagnetics Group, Bilkent University. From August 2001 to December 2006, he was a Research Assistant at the Center for Computational Electromagnetics and Electromagnetics Laboratory, UIUC. From January 2007 to August 2009, he worked as a Research Fellow at the Radiation Laboratory, University of Michigan. In August 2009, he joined the Division of Physical Sciences and Engineering at the King Abdullah University of Science and Technology (KAUST) as Assistant Professor of Electrical Engineering. His research interests include various aspects of computational electromagnetics with emphasis on timedomain integral equations and their fast marching-on-in-time-based solutions, well-conditioned integral-equation formulations, and development of fast hybrid methods for analyzing statistical EMC/EMI phenomena on complex and fully loaded platforms. Dr. Bağcı was the recipient of the 2008 International Union of Radio Scientists (URSI) Young Scientist Award and the 2004-2005 Interdisciplinary Graduate Fellowship from the Computational Science and Engineering Department, UIUC. His paper titled "Fast and rigorous analysis of EMC/EMI phenomena on electrically large and complex structures loaded with coaxial cables" was one of the three finalists (with honorable mention) for the 2008 Richard B. Schulz Best Transactions Paper Award given by the IEEE Electromagnetic Compatibility Society. He authored and co-authored five finalist papers in the student paper competitions at the 2005, 2008, and 2010 IEEE Antennas and Propagation Society International Symposiums and 2013 Applied Computational Electromagnetics Society Conference. 